\title{
A pilot study assessing the various dermatoses associated with the use of a face mask during the COVID-19 pandemic
}

\section{Nagaria Nishi, Sonappa Uday Kumar, Talari Srinivas Rajashekar, Keloji Hanumanthayya, Kuppuswamy Suresh Kumar}

\author{
Department of Dermatology, Sri Devaraj Urs Medical College, Tamaka, Kolar, India
}

Corresponding author: Sonappa Uday Kumar, MD, E-mail: udnvab@gmail.com

\begin{abstract}
Background: An occupational disease is any disease developing primarily as a result of exposure to risk factors arising from work activity and contributing to a significant portion of work-related diseases. Although COVID-19 is not dermatotropic, prolonged contact with personal protective equipment— that is, goggles, face shields, N95 respirators, double-layered gloves, etc.-may cause various dermatoses. Several dermatoses due to PPE have been well documented, but facial dermatoses specifically due to the use of face masks remain a relatively uncharted entity. In this study, we report preliminary data on individuals experiencing various facial dermatoses due to the use of face masks. Objective: The aim was to study the various facial dermatoses associated with the use of a face mask. Materials and Methods: After obtaining informed consent, individuals using a face mask were enrolled in this single-institution, questionnaire-based, cross-sectional study. Results: A total of 364 participants were enrolled for the study, among which 59.3\% revealed to have worn a face mask for more than six months. A majority used ear-looped N95 masks. Nine problem areas were identified in the study. Among the facial dermatoses observed, acne was the most frequently reported, followed by redness. Conclusion: In spite of contact dermatitis arising due to masks, it is recommended to use well-fitted and comfortable masks for sufficient periods of time to reduce the risk of transmission, ensure safety, and reduce maskrelated side effects.
\end{abstract}

Key words: Dermatitis; Mask-induced; Facial dermatoses; COVID-19; Mask dermatitis

\section{INTRODUCTION}

SARS-CoV-2, the COVID-19 virus, or the novel coronavirus are the various names given to the virus that originated in December 2019 in a small town in China called Wuhan [1]. Being highly infectious, it affected hundreds of thousands of people within a short amount of time worldwide, causing the World Health Organization to announce it as an infectious disease pandemic on January 30, 2020 [2].

The COVID-19 virus is spread by droplets or via fomites, creating the necessity to wear personal protective equipment, even if not directly in contact with patients affected by COVID-19 [1].
The mucosal membrane is the most common portal of entry for infection, including the conjunctiva and the optic canal-although with the lowest risk of transmission. Therefore, specific skin changes due to the COVID -19 virus are because of the secondary iatrogenic involvement of the skin [3].

An occupational disease is any disease that develops primarily due to work-related exposure to risk factors. Among all conditions coming under the wide umbrella of occupational dermatoses, the most common is occupational contact dermatitis, accounting for the majority of cases (79-95\%), followed by contact urticaria, occupational marks, infectious dermatoses, and neoplasia, observed only in some [4].

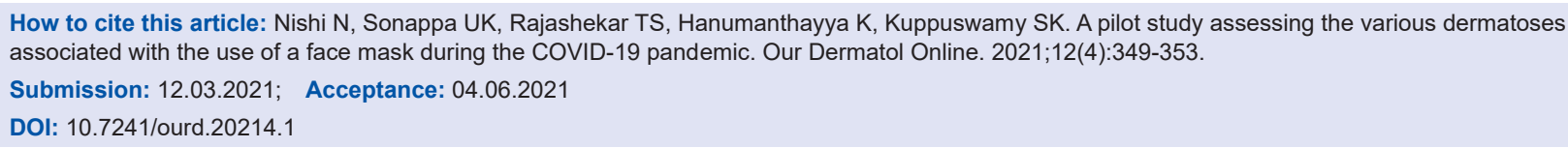


PPE includes gowns, gloves, masks, shoe covers, head covers, eye gear, and face shields [5]. Apart from the major shortage of PPE for healthcare professionals on the front lines and for the general public, the adverse effects from the long use of PPE are another major cause of concern [6].

According to the CDC and the WHO, wearing a face mask is mandatory if the patient is suffering from highly transmissible diseases such as tuberculosis, SARS, and COVID-19. The mask recommended for use is the N95 mask, with the N standing for NIOSH (National Institute for Occupational Safety and Health of the United States) and 95 indicating the filter efficiency of the particles. This, in simpler terms, means that an N95 mask is capable of effectively filtering $95 \%$ of airborne particles, including very small [7]. This is in comparison to the widely used surgical masks, which provide a barrier against large respiratory particles, but are ineffective in providing protection against smaller particles. Also, surgical masks are inefficient in preventing leakage around the mask during inhalation. Therefore, the reason why N95 masks are recommended is that surgical masks are ineffective and do not provide enough protection to people involved in direct care of patients with COVID-19 [2].

Among all measures taken to prevent the spread of COVID-19, the most important is social distancing, proper sanitization of the hands, and the use of face masks in and around the hospitals, and even inside the house.

Long hours of wearing a face mask and PPE come with a plethora of problems of their own, ranging from physiological to psychological burdens, and may also decrease the user's work efficiency. Prolonged use of N95 and surgical masks causes adverse effects such as difficulty in breathing, headaches, acne, rashes, skin breakdowns, and impaired cognition, to name a few [2]. Sensitivity to components in masks and PPE may cause urticarial and contact dermatitis. The most common chemical used in PPE is formaldehyde, which causes sensitivity and allergy to a significant portion of the population. Several others may also react with thiuram, found in the ear loops of surgical masks [8].

The COVID-19 pandemic has forged an exponential use of face masks of various kinds as PPE, not only by health workers but also by the general population [9].

Although contact dermatitis due to PPE has been well reported, mask-induced dermatitis is a relatively unexplored phenomenon, which is why this study was conducted, namely, to report preliminary data on individuals experiencing various facial dermatoses due to face masks.

\section{MATERIALS AND METHODS}

This anonymous, single-center, cross-sectional, questionnaire-based survey was distributed electronically at the beginning of November 2020 to healthcare professionals, patients, and their attenders. The majority of the survey respondents were males $(51.1 \%, n=186)$. The respondents' age ranged from less than 20 to more than 60 years. $3.6 \%(n=13)$ were less than 20 years old. $69.2 \%(n=252)$ were aged between 20 and 40 years, 26.4\% $(n=96)$ between 40 and 60 , and $0.80 \%(n=3)$ were 60 years old and older.

\section{Ethics Statement}

The questionnaire and methodology of this study were approved by the Institutional Ethical Committee (ethics approval number: No. DMC/KLR/IEC/412/2020-21).

\section{RESULTS}

Among the 364 participants of this survey, all consented to participate in the study. 361 respondents $(99.2 \%)$ agreed to wearing a mask (Fig. 1$)$, with $59.3 \%(n=216)$ having worn a mask for more than six months, $35.2 \%$ $(n=128)$ claiming to have worn a mask for 3-6 months, and about $5.5 \%(n=20)$ having worn a mask for less than three months (Fig. 2). Most (53.02\%, $n=193$ ) of the interviewees have worn a mask for 6-12 hours daily, whereas $40.4 \%(n=147)$ did so for less than six hours, and $6.6 \%(n=24)$ for more than twelve hours (Fig. 3). A majority $(69.8 \%, n=254)$ of the mask users have worn an N95 mask, followed by $38.7 \%(n=141)$ of those wearing surgical masks, $34.6 \%(n=126)$ fabric masks, $8.5 \%(n=31) \mathrm{FFP}$, and $7.1 \%(n=26)$ something yet different (Fig. 4). Most of the mask users used ear-looped masks $(77.5 \%, n=282)$ and $49.7 \%$ $(n=181)$ used head-looped masks. The study revealed that $76.4 \%(n=278)$ of the respondents who used masks had regularly experienced facial dermatitis in one or other form, while $23.6 \%(n=86)$ reported none of these side effects (Fig. 5). In the study, pimples were one of the most prevalent skin reactions related to the use of a face mask. Other reported side effects included breathlessness $(28.8 \%, n=105)$, redness $(28.8 \%$, $n=105)$, rashes $(25.5 \%, n=93)$, itching $(23.6 \%$, 
$n=86)$, oily skin $(21.7 \%, n=79)$, bad breath $(17.6 \%$, $n=64)$, blocked nose $(15.1 \%, n=55)$, and raw skin $(10.4 \%, n=38)$, to name a few (Fig. 5). Among 31.9\%

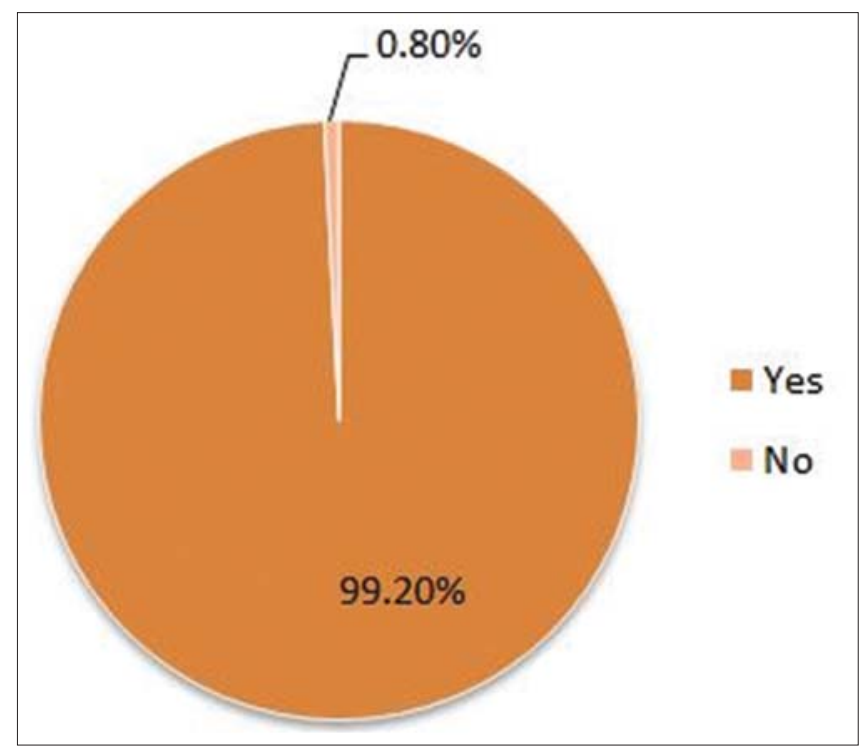

Figure 1: Do you wear a mask?.

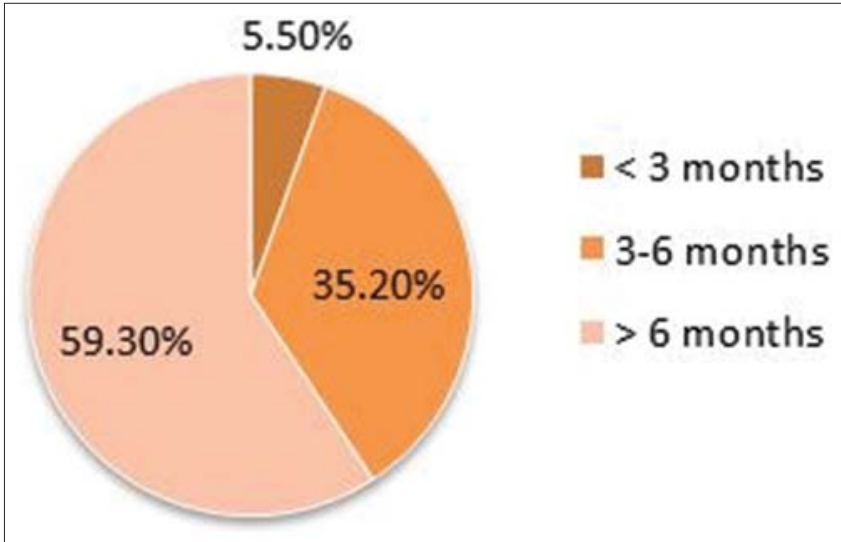

Figure 2: Duration (From when the mask is being used).

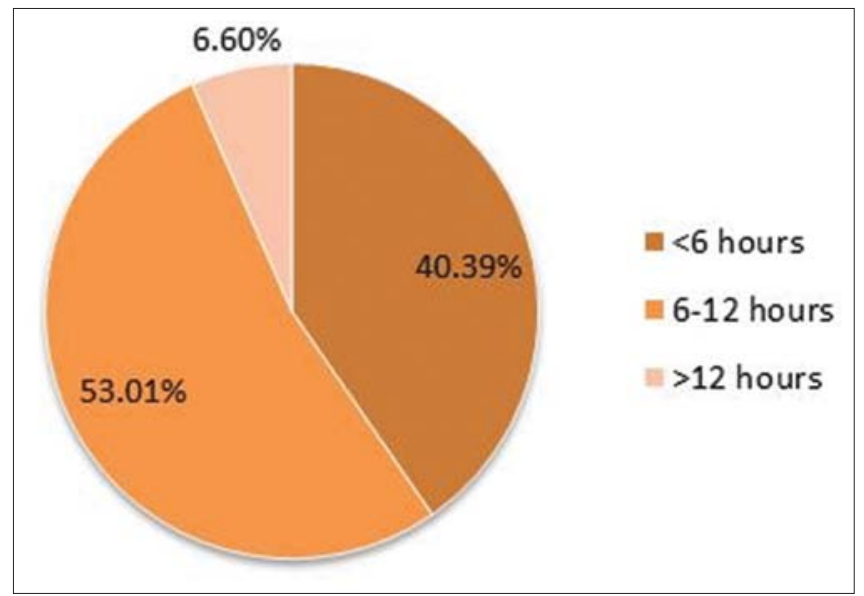

Figure 3: Number of hours of using mask daily of the survey respondents $(n=116)$ reporting pimples as an adverse effect, $73.7 \%(n=140)$ complained of papules while $36.3 \%(n=69)$ complained of pustules. For those who had had adverse skin effects, the most common area was the bridge of the nose $(31.6 \%$, $n=115)$ and the cheeks $(26.9 \%, n=98)$. Other areas of skin breakdown were the chin $(19.8 \%, n=72)$, the area behind the ears $(17 \%, n=62)$, the jawline $(17.7 \%$, $n=41)$, and the nape of the neck $(4.1 \%, n=15)$ (Fig. 6).

\section{DISCUSSION}

The COVID-19 pandemic has spread rapidly across the globe, greatly affecting how people as a whole interact,

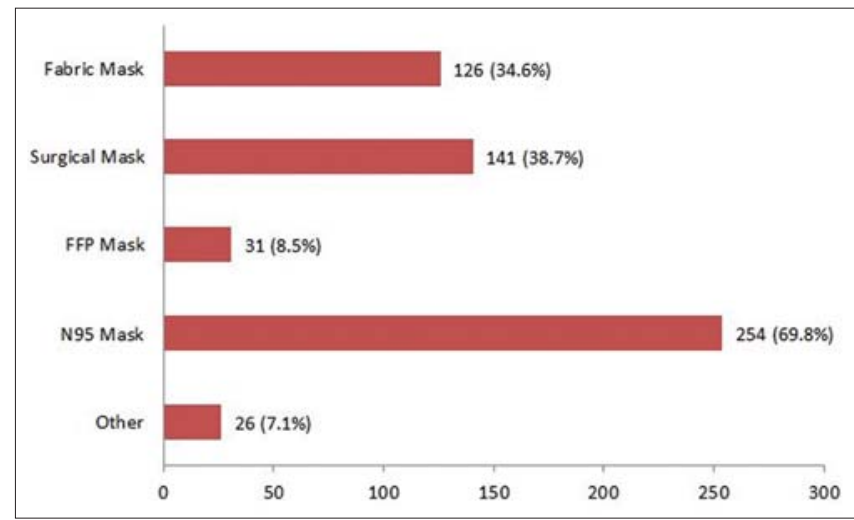

Figure 4: Type of mask used.

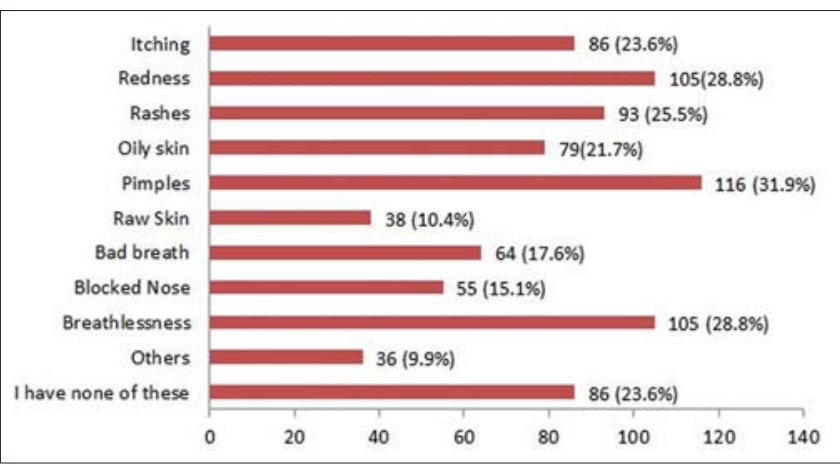

Figure 5: Main issue faced by the mask user.

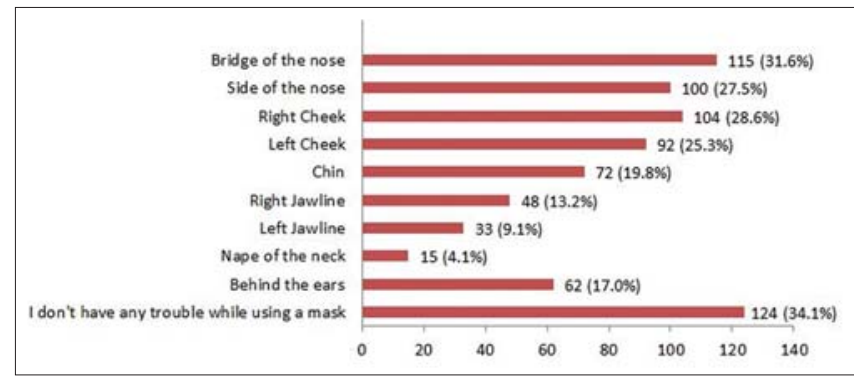

Figure 6: Areas of trouble. 
work, and go about their daily life [1]. We are still in the very middle of the pandemic, and it does not seem to be ending any time soon. In fact, the cases are again on the rise, pointing toward a second wave [2].

Appropriate personal protective equipment (PPE) and frequent hand hygiene are suggested to prevent transmission of the virus [10]. However, there is evidence suggesting that these practices have a negative impact on skin health [11].

A great number of subjects who participated in this study reported adverse reactions to prolonged mask use during COVID-19. Pimples, breathlessness, rashes, and redness were all recognized as common adverse effects, which was in accordance with a study by Rosner [2]. A former study indicated that more than one-third of health care workers complained of acne, facial itching, and even dermatitis from wearing an N95 mask [12].

The skin complications are due to the hyperhydration effect of personal protective equipment (PPE), friction, epidermal barrier breakdown, and contact reactions, all of which may aggravate an existing skin disease [12].

Wearing properly fitted masks, avoiding latex straps, using soft materials, frequently changing one's masks, taking regular breaks to remove one's mask, wiping the skin to remove sweat, and frequently washing the face may help to alleviate these dermatoses [13].

Preventive measures such as removing one's mask for 10-15 minutes every two hours, provided it is safe to do so, using a gentle, non-comedogenic, fragrancefree cleanser twice a day, ensuring that the area is free of makeup, wearing straps on the crown of the head instead of straps sitting on top of the ears, and applying an alcohol-free barrier film on the areas of direct contact with PPE-for instance, the nose, cheeks, and the area behind the ears-to protect the skin from unnecessary friction may tackle most of the problems. Applying petrolatum to open areas 3-4 times a day if skin damage is present will be beneficial. For open areas of skin damage, a hydrocolloid dressing may help in faster healing; however, using these dressings under an N95 mask requires refit testing to ensure adequate seal [13].

The authors suggest using homemade cotton-cloth face masks by the general population while at home. Those with preexisting dermatoses such as chronic urticaria, seborrheic dermatitis, or atopic dermatitis should take special precautions, and the use of disposable surgical masks should be encouraged [8].

\section{CONCLUSION}

At present, there is widespread use of facial masks due to the COVID-19 pandemic, which has led to an increase in the incidence of allergies and contact dermatitis caused by contact with face masks [14]. Improved hydration and rest, skincare, frequent breaks, and properly fitted, comfortable masks are recommendations for future management of the adverse effects related to prolonged mask use [2].

\section{Limitations}

While this survey captured the experiences of the general population, including many health care professionals or those associated with the healthcare facility working on the front lines during COVID-19, there are some limitations to this study. First, preexisting conditions such as a high BMI, asthma, and other conditions were not assessed in this survey, and these could be impacting or increasing the adverse effects addressed in this survey. Second, issues such as high stress levels and the quality of sleep were also not included in this survey, and these important factors may also contribute to the adverse effects reported by the survey's respondents.

\section{Statement of Human and Animal Rights}

All the procedures followed were in accordance with the ethical standards of the responsible committee on human experimentation (institutional and national) and with the 2008 revision of the Declaration of Helsinki of 1975.

\section{Statement of Informed Consent}

Informed consent for participation in this study was obtained from all patients.

\section{REFERENCES}

1. O'Dowd K, Nair KM, Forouzandeh P, Mathew S, Grant J, Moran R, et al. Face masks and respirators in the fight against the COVID-19 pandemic: A review of current materials, advances and future perspectives. Materials (Basel). 2020;13:3363.

2. Rosner E. Adverse effects of prolonged mask use among healthcare professionals during COVID-19. J Infect Dis Epidemiol [Internet]. 2020;6:130.

3. Darlenski R, Tsankov N. COVID-19 pandemic and the skin: What should dermatologists know? Clin Dermatol. 2020;38:785-7.

4. Sharma V, Bhatia R. Occupational dermatoses: An Asian perspective. Indian J Dermatol Venereol Leprol. 2017;83:525-35 
5. Cook TM. Personal protective equipment during the coronavirus disease (COVID) 2019 pandemic - A narrative review. Anaesthesia. 2020;75:920-7.

6. Foo CC, Goon AT, Leow YH, Goh CL. Adverse skin reactions to personal protective equipment against severe acute respiratory syndrome-A descriptive study in Singapore. Contact Dermatitis. 2006;55:291-4.

7. Center for Disease Control and Prevention (2020) NIOSHapproved N95 particulate filtering facepiece respirators: Ancillary respirator information

8. Bothra A, Das S, Singh M, Pawar M, Maheswari A. Retroauricular dermatitis with vehement use of ear loop face masks during COVID-19 pandemic. J Eur Acad Dermatol Venereol. 2020;34:e549-52.

9. World Health Organization. Infection prevention and control during health care when novel coronavirus ( $\mathrm{nCoV}$ ) infection is suspected. 2020. Available at: https://www.who.int/ publications-detail/ infection-prevention-and-controlduring-health-care-when-novelcoronavirus-(ncov)- infection-is-suspected-20200125 (accessed 10 June 2020)

10. Kiely LF, Moloney E, O’Sullivan G, Eustace JA, Gallagher J, Bourke JF. Irritant contact dermatitis in healthcare workers as a result of the COVID-19 pandemic: A cross-sectional study. Clin Exp Dermatol. 2021;46:142-4.
11. Elston DM. Occupational skin disease among health care workers during the coronavirus (COVID-19) epidemic. J Am Acad Dermatol. 2020;82:1085-6.

12. Singh M, Pawar M, Bothra A, Maheshwari A, Dubey V, Tiwari A, Kelati A. Personal protective equipment induced facial dermatoses in healthcare workers managing Coronavirus disease 2019. J Eur Acad Dermatol Venereol. 2020;34:e378-80.

13. Desai SR, Kovarik C, Brod B, James W, Fitzgerald ME, Preston A, et al. COVID-19 and personal protective equipment: Treatment and prevention of skin conditions related to the occupational use of personal protective equipment. J Am Acad Dermatol. 2020;83:675-7.

14. Lin P, Zhu S, Huang Y, Li L, Tao J, Lei T, et al. Adverse skin reactions among healthcare workers during the coronavirus disease 2019 outbreak: A survey in Wuhan and its surrounding regions. $\mathrm{Br}$ J Dermatol. 2020;183:190-2.

Copyright by Nagaria Nishi, et al. This is an open access article distributed under the terms of the Creative Commons Attribution License, which permits unrestricted use, distribution, and reproduction in any medium, provided the original author and source are credited.

Source of Support: Nil, Conflict of Interest: None declared. 